\title{
Maternal and perinatal outcome in preterm premature rupture of membranes
}

\section{Jameela Diraviyam M. V.*, Lalithambica Karunakaran}

Department of Obstetrics and Gynecology, Government T D Medical College, TDMCH, Alappuzha, Kerala, India

Received: 30 March 2017

Accepted: 27 April 2017

\section{* Correspondence:}

Dr. Jameela Diraviyam M. V.,

E-mail: mvjameela@gmail.com

Copyright: (c) the author(s), publisher and licensee Medip Academy. This is an open-access article distributed under the terms of the Creative Commons Attribution Non-Commercial License, which permits unrestricted non-commercial use, distribution, and reproduction in any medium, provided the original work is properly cited.

\section{ABSTRACT}

Background: Preterm premature rupture of membranes (PPROM) occurs in 3\% of pregnancies and is responsible for approximately one third of all preterm births. Objective of present study was to analyse the maternal and perinatal outcome of PPROM patients between 28 to 36 weeks +6days

Methods: A descriptive study was conducted on 141 antenatal patients between 28 to 36weeks+6days with PPROM admitted to Department of Obstetrics and Gynecology, Government TD Medical College, Alappuzha, Kerala, India from September 2014 to September 2015. After establishing the diagnosis of PPROM patients were monitored and Maternal and perinatal outcomes were studied.

Results: $77 \%$ patients had late PPROM. $60 \%$ of early PPROM latency period $>24$ hrs and were managed conservatively till 34 weeks. $18 \%$ had chorioamnionitis and immediate termination of pregnancy. $73 \%$ of newborns in this group needed admission due to complications of prematurity like RDS (54.54\%). Perinatal mortality (2.12\%) was due to sepsis. $80 \%$ of late PPROM had latency period $<24 \mathrm{hrs}$ and only $4 \%$ had chorioamnionitis. $18.5 \%$ babies in this group had hyperbilirubinemia. There was statistically significant association between latency period and perinatal complications ( $\mathrm{p}=0.001$ ). RDS was $33 \%$ in latency period $<24 \mathrm{hrs}, 18 \%$ in $>24 \mathrm{hrs}$ and sepsis was $36 \%$ in $>24 \mathrm{hrs}$ and $10 \%$ in $<24 \mathrm{hrs}$.

Conclusions: The most common cause of perinatal mortality in early PPROM is prematurity and its complications. Hence conservative management to prolong pregnancy is recommended under strict monitoring for evidence of chorioamnionitis. At the earliest evidence of chorioamnionitis termination irrespective of gestational age is warranted. In late PPROM, perinatal outcome is good. So, termination is advised as conservative management shall add to the fetal and maternal morbidity due to sepsis.

Keywords: Choriomnionitis, Latency period, Maternal outcome, Perinatal outcome, Sepsis

\section{INTRODUCTION}

Preterm premature rupture of membranes (PPROM) occurs in $3 \%$ of pregnancies and is responsible for approximately one third of all preterm births (Bartfield 1998; Goldenberg 1998). ${ }^{1,2}$ The incidence of preterm premature rupture of membrane averages from 0.7 to $2.1 \%$ and accounts for about 20 to $40 \%$ cases of PROM before 37 weeks of gestation. Jayaram et al analysed 100 patients with PROM between 32-40 weeks, and found the incidence of PROM to be $7.71 \%(69 \%$ term and $31 \%$ PPROM). ${ }^{3}$

Although preterm premature rupture of membrane complicates about 2-4\% of singleton pregnancies and 7$20 \%$ of twin pregnancies, it is associated with $60 \%$ preterm deliveries and $10 \%$ of perinatal death. Preterm PROM is an important cause of perinatal morbidity and 
mortality, particularly because it is associated with brief latency from membrane rupture to delivery, perinatal infection, and umbilical cord compression due to oligohydramnios.

Preterm premature rupture of membranes is defined as spontaneous rupture of amniotic membranes before the onset of uterine contractions or prior to the onset of labour after the age of viability and before 37 completed weeks (36weeks+6days). Latency period is the time interval between the rupture of the membranes and the onset of uterine contractions. Kappy and Khupel defined PROM as rupture of the membranes with atleast 2 hours of latent period before active labour. ${ }^{4}$ The membranes may either rupture at term ( $>37$ weeks) when it is called term PROM or before 37 weeks of gestation when it is refered to as preterm PROM (PPROM).

PPROM is associated with increased risk of chorioamnionitis, unfavourable cervix, dysfunctional labour, increase in caesarean rates, postpartum hemorrhage and endometritis in mother. In fetus increased occurrence of hyaline membrane disease, intraventricular hemorrhage, sepsis, cord prolapse, fetal distress and increased fetal wastage. The longer the time interval between the rupture of membranes and onset of labour, greater is the risk of ascending infections and chorioamnionitis. This risk may assume grave proportions in patient undergoing ceaserean section. Thus, earlier the gestational age at the time of PPROM, longer the latency and more the complications.

In planning the management of PPPROM, several issues need to be considered. Prematurity is the principal risk to the fetus while infectious morbidity is the primary maternal risk. Chorioamnionitis with PPROM is responsible for significant maternal and neonatal morbidity including early onset neonatal sepsis, bronchopulmonary dysplasia, intraventricular haemorrhage and periventricular white matter injury. PPROM is an obstetric conundrum with significant maternal morbidity and neonatal morbidity and mortality, a careful consideration of various factors and individualization of cases is necessary for appropriate management.

Objective of present work was to study the maternal and perinatal outcome in women admitted with preterm premature rupture of membranes (PPROM), between 28 weeks and 36weeks+6days, in labour room of Obstetrics and Gynecology Department, in Govt. T.D. Medical college Alappuzha, Kerela from September 2014 to September 2015.

\section{METHODS}

It is a descriptive study conducted at Government TDMC Alappuzha. Samples were those who got admitted with PPROM in labour room between 28weeks and 36weeks+6days. The diagnosis of PPROM was established by history, sterile pelvic speculum examination showing amniotic fluid trickling from cervix, pad test. Ultrasonography was done in each case to asses gestational age, growth parameters, presentation, exclusion of congenital anomalies and liquor columns for amniotic fluid index. USG was done mainly in those patients who were treated conservatively.

Conservative management was done in all early PPROM (28weeks to 33 weeeks+6days) patients till the onset of spontaneous labour or till the maternal or fetal indication for delivery ensues such as chorioamnionitis, meconium stained amniotic fluid, abruption, cord prolapse, fetal distress and/or advanced labour on admission. All late PPROM (>34weeks) patients were induced if not getting into spontaneous labour. Patients were hospitalized until delivery \& were advised bed rest. Two doses of betamethasone $12 \mathrm{mg}$ I.M 12 hours apart were given to the mothers <34weeks to enhance fetal lung maturity. Prophylactic antibiotics were used in all cases for ten days or up to delivery (whichever is later) to reduce the risk of infection. Maternal monitoring to detect chorioamnionitis was done by monitoring pulse rate, temperature, abdominal tenderness, color and smell of liquor and cardiotocography C.T.G.

Mothers were monitored intrapartum for complications such as abruption, PPH, retained placenta. Neonates with poor APGAR score or infection were admitted in IBN (in born nursery) for further management and their outcome were studied. Mother and babies were followed up till discharge.141 cases were studied, data analyzed and expressed in its frequency and percentage.

\section{RESULTS}

Of 141 patients studied ,77\% had late PPROM and $23 \%$ had early PPROM (Table 1). 94.32\% were singleton pregnancies, $4.25 \%$ twins, $1.04 \%$ triplets (Table 2).

Table 1: Distribution of early and late PPROM.

\begin{tabular}{|l|c|}
\hline & $\%$ \\
\hline Early PPROM & 23 \\
\hline Late PPROM & 77 \\
\hline
\end{tabular}

Table 2: Distribution of singleton and multiple pregnancies in study population.

\begin{tabular}{|ll|}
\hline Singleton & $\%$ \\
\hline Twins & 94 \\
\hline Triplets & 4 \\
\hline
\end{tabular}

The early PPROM was common in 26 to $30 \mathrm{yrs}$, whereas late PPROM in 21 to $25 \mathrm{yrs}$ (Table 3). Most of them fall under lower socioeconomic status. Almost all were booked cases. Most of the PPROM patients in the present study were primigravida $63.12 \%$ (Table 4). Both early and late PPROM were common among primigravida. 
Table 3: Age distribution.

\begin{tabular}{|ll|}
\hline Age & $\%$ \\
\hline$<20 y r s$ & 11 \\
\hline $20-25 y r s$ & 41 \\
\hline $25-30 y r s$ & 31 \\
\hline $30-35 y r s$ & 13 \\
\hline$>35 y r s$ & 4 \\
\hline
\end{tabular}

Table 4: Distribution of obstetric score in study population.

\begin{tabular}{|ll|}
\hline Obsteric score & $\%$ \\
\hline Primi & 63.12 \\
\hline G3 & 32.62 \\
\hline
\end{tabular}

Majority of PPROM in multigravida were associated with no significant past obstetric history, those with past history 29\% had abortion, 13\% had PPROM, 10\% had preterm delivery.

Table 5: Distribution of latency period.

\begin{tabular}{|ll|l|}
\hline Latency period & Early PPROM & Late PPROM \\
\hline$<24 \mathrm{hrs}$ & $40 \%$ & $80 \%$ \\
\hline$>24 \mathrm{hrs}$ & $60 \%$ & $20 \%$ \\
\hline
\end{tabular}

$60 \%$ of early PPROM (28weeks-33weeks 6days) and $20 \%$ of late PPROM (34weeks-36weeks 6days) had prolonged latency period (>24hrs) (Table 5). The induction rate was more common in patients with prolonged latency period. Most of the early and late PPROM patients delivered vaginally, only $21 \%$ had LSCS. The most common indication for primary LSCS in PPROM was malpresentations, followed by fetal distress and failed induction.

Table 6: Distribution of maternal morbidity.

\begin{tabular}{|lll|}
\hline $\begin{array}{l}\text { Maternal } \\
\text { complications }\end{array}$ & $\begin{array}{l}\text { Early } \\
\text { PPROM }\end{array}$ & Late \\
\hline Chorioamnionitis & $18 \%$ & $4 \%$ \\
\hline Abruption & $6 \%$ & $0 \%$ \\
\hline No complications & $76 \%$ & $96 \%$ \\
\hline
\end{tabular}

Most of patients in early and late PPROM had no complications. Complications like chorioamnionitis and abruption were observed in patients with prolonged latency period. Of $60 \%$ of early PPROM with prolonged latency $18 \%$ had chorioamnionitis and $6 \%$ had abruption, $20 \%$ of late PPROM with prolonged latency $4 \%$ had chorioamionitis (Table 6 ) of the babies born out of PPROM $59 \%$ were males, $41 \%$ were females.

About $73 \%$ of newborn in early PPROM $27 \%$ late PPROM needed IBN admission because of prematurity and associated complications. The mean birth weight of babies in early PPROM was $1.8 \mathrm{~kg}$ and in late PPROM was $2.45 \mathrm{~kg}$. In early PPROM commmom complication were RDS and sepsis accounting for $54.54 \%$ and $12.12 \%$ respectively (Table 7).

Table 7: Distribution of perinatal morbidity.

\begin{tabular}{|lll|}
\hline $\begin{array}{l}\text { Perinatal } \\
\text { complications }\end{array}$ & Early & Late \\
\hline Sepsis & $12.12 \%$ & $3.70 \%$ \\
\hline RDS & $54.54 \%$ & $9.25 \%$ \\
\hline NEC & $6 \%$ & $0 \%$ \\
\hline Hyperbilirubinemia & $24.24 \%$ & $18.51 \%$ \\
\hline
\end{tabular}

In the present study RDS was $33 \%$ in $<24 \mathrm{hrs}, 18 \%$ in $>24$ hrs, sepsis was more about $36 \%$ in $>24 \mathrm{hrs}$ and $10 \%$ in $<24 \mathrm{hrs}$, Hyperbilirubinemia was $46 \%$ in $<24 \mathrm{hrs}, 45 \%$ in $>24 \mathrm{hrs}$, NEC was $3 \%$ in $<24 \mathrm{hrs}$ and no NEC in $>24 \mathrm{hrs}$.

Table 8: Latency period VS perinatal morbidity.

\begin{tabular}{|lll|}
\hline Perinatal & $\begin{array}{l}\text { Latency } \\
\text { complications }\end{array}$ & $\begin{array}{l}\text { Latency } \\
>24 h \mathrm{hrs}\end{array}$ \\
\hline Sepsis & $19 \%$ & $3.70 \%$ \\
\hline RDS & $54.54 \%$ & $9.25 \%$ \\
\hline NEC & $6 \%$ & $0 \%$ \\
\hline Hyperbilirubinemia & $24.24 \%$ & $18.51 \%$ \\
\hline
\end{tabular}

The cause of mortality in the present study was sepsis. All of them were between 28 weeks-29 weeks and latency period of $<24 \mathrm{hrs}$ (Table 8 ). Sepsis was the cause of neonatal mortality in our study (Table 9).

Table 9: Perinatal mortality.

\begin{tabular}{|c|c|c|c|}
\hline $\begin{array}{l}\text { Geatational } \\
\text { age }\end{array}$ & $\begin{array}{l}\text { Latency } \\
\text { period }\end{array}$ & $\begin{array}{l}\text { Birth } \\
\text { weight }\end{array}$ & $\begin{array}{l}\text { Cause of } \\
\text { mortality }\end{array}$ \\
\hline 29weeks & $12 \mathrm{hrs}$ & $1.3 \mathrm{~kg}$ & $\begin{array}{l}\text { Sepsis, pulmonary } \\
\text { hemorrhage }\end{array}$ \\
\hline 28weeks & $6 \mathrm{hrs}$ & $1 \mathrm{~kg}$ & Sepsis, DIC \\
\hline 28weeks & $5 \mathrm{hrs}$ & $1 \mathrm{~kg}$ & Still born \\
\hline
\end{tabular}

\section{DISCUSSION}

In present study $41 \%$ were between $21-25 y$ rs of age. In a study by Noor S et al in Ayub Medical College in 2006, $58.8 \%$ were in the age group of 21-25yrs. ${ }^{5}$ Among 141 patients, $67 \%$ of them belong to lower socioeconomic status. In a study done by Sheela et al $68.2 \%$ were under lower socioeconomic group. ${ }^{5}$ Among the total study population $63.12 \%$ were primigravida. In a study conducted by Ghandhi $\mathrm{M}$ et al $60.7 \%$ were primigravida, another study by Sheela et al majority were primigravida $42.3 \%$, another study by Okeke et al majority were primigravida $29.1 \% .^{5-7}$ In present study $48 \%$ of multigravida had no significant past history. 29\% had abortions, $13 \%$ had PPROM. In a study by Revathi et al $17 \%$ had previous abortion, $10 \%$ had previous PPROM. ${ }^{8}$ Another study by Sheela et al $29 \%$ had previous preterm deliveries, in the present study $10 \%$ had previous preterm deliveries. $^{5}$ 
In our study $60 \%$ of early PPROM and $20 \%$ of late PPROM had prolonged latency period. Singal $S$ et al study showed $56 \%$ of early PPROM and $20 \%$ of late PPROM had prolonged latency. ${ }^{9}$ In a study by Gunvat et al $44 \%$ of late PPROM had prolonged latency. ${ }^{10}$ In early PPROM $70 \%$ had vaginal delivery, $30 \%$ underwent LSCS, in late PPROM $81 \%$ vaginal delivery and $19 \%$ LSCS. In a study by Sheela et al $65 \%$ had vaginal $15 \%$ had LSCS, Biwas $\mathrm{T}$ et al $72 \%$ had vaginal $16 \%$ had LSCS. In the present study $40 \%$ of LSCS were among indicated deliveries, a study by Jenny et al showed $18.8 \%$ LSCS rate in indicated deliveries. ${ }^{11}$ LSCS rate were high in the present study because according to our institution protocol breech presentations were not kept for vaginal delivery.

Chorioamnionitis was more common in indicated deliveries as they had prolonged latency period and they were prone to infection during this period. In a study by Bryden et al also showed $67 \%$ of chorioamnionitis among early PPROM were in indicated delivery. ${ }^{12}$ Another study by Jenny et al showed $1.6 \%$ of chorioamnionitis in late PPROM. ${ }^{11}$ In the present study (16\%) had abruption in early PPROM, no abruption in late PPROM.

Major neonatal morbidity noted in our study was RDS contributing to $54.5 \%$ followed by $24.2 \%$ by hyperbilirubinemia, $12.1 \%$ by sepsis, and $6 \%$ by necrotizing enterocolitis. RDS is more common in early PPROM and hyperbilirubinemia was more common in late PPROM. A study by Emeche et al showed 61\% RDS, Singal $\mathrm{S}$ et al obtained $92 \%$ RDS in early PPROM. ${ }^{13} \mathrm{~A}$ study by Emeche et al showed $22 \%$ RDS, 17.8 hyperbilirubinemia, and $16 \%$ sepsis. ${ }^{13}$ Similar study by Singal S et al, in late PPROM showed 6.6\% RDS, $16 \%$ sepsis and $25 \%$ NEC. In present study, $9.2 \%$ had RDS, $18.5 \%$ had hyperbilirubinemia, $3.7 \%$ had sepsis.

In present study, RDS was more common in patients who delivered within $24 \mathrm{hrs}$, and sepsis was common in patients who had prolonged latency period. $33 \%$ had RDS, $2.5 \%$ had NEC and 10\% had sepsis in patients whose who delivered within 24 hrs. In a Similar study by Singal S et al, $46.6 \%$ had RDS, and $25 \%$ had NEC. ${ }^{9}$ Among patients who had prolonged latency period of more than $24 \mathrm{hrs}, 18 \%$ had RDS $36 \%$ had sepsis, and $0 \%$ NEC. In a similar study by seema et al, 33.3\% had RDS and $50 \%$ had NEC. Perinatal mortality was $2.12 \%$ in this study, whereas $7.6 \%$ mortality in a study by Seema et al. Early PPROM mainly contributes to perinatal mortality. Sepsis was the main cause of perinatal mortality.

\section{CONCLUSION}

Present study concluded that most common maternal morbidity associated with PPROM was chorioamnionitis, that of neonatal morbidity was prematurity and its complications. In current study, of the patients with PPROM most of them were between 2125years.Incidence of PPROM was more common in primigravidas. Most multigravida had no significant past obstetric history, of those with past history most of them had abortions. More women had spontaneous delivery, inductions were common in late PPROM. Most of the patients delivered vaginaly, LSCS were done commonly for malpresentations like breech, followed by fetal distress and failed inductions.

Common maternal morbidity observed in present study was chorio amnionitis. It was more common among the patients with prolonged latency and induced deliveries. Perinatal morbidity observed in our study were due to prematurity and not the PPROM per se. commonest complication encountered was RDS which was more common in early PPROM. Other complications like neonatal jaundice, sepsis was more or less equally common among early and late PPROM.

Sepsis was more common in patients with prolonged latency period, also contributes to perinatal mortality, whereas RDS was more common in patient with short latency period.

In early PPROM conservative management to prolong pregnancy is recommended under strict monitoring for evidence of chorioamnionitis. At the earliest evidence of chorioamnionitis termination irrespective of gestational age is warranted. In late PPROM termination is advised as conservative management shall add to the fetal and maternal morbidity due to sepsis

\section{ACKNOWLEDGMENTS}

Authors would like to acknowledge the faculties and other staff members of the Department of OBG, Government TD medical college, Alappuzha in their respective assistance in preparing this manuscript.

Funding: No funding sources Conflict of interest: None declared

Ethical approval: The study was approved by the Institutional Ethics Committee

\section{REFERENCES}

1. Bartfield MC, Carlan SJ. The home management of preterm premature ruptured membranes. Clini Obstet Gynecol. 1998;41(3):503-14.

2. Goldenberg RL, Rouse DJ. Prevention of premature birth. N Eng J Med. 1998;339(5):313-20.

3. Jayaram VK, Sudha S. A study of PROM: management and outcome. J Obstet Gynecol India. 2001;51:58-60.

4. Khuppel Ka, Curtis C, Robert LK. Premature rupture of membranes. Am J Obstet Gynecol. 1979;134(6):655-61.

5. Noor S, Nazar AF, Bashir R, Sultana R. Prevalance of PPROM and its outcome. J Ayub Med Coll Abbottabad. 2007;19(4):14-7. 
6. Gandhi M, Shah F, Panchal C. Obstetric outcomes in premature rupture of the membrane (Prom). Internet J Gynecol Obstet. 2012;16(2):1-5.

7. Okeke TC, Enwereji JO, Okoro OS, Adiri CO, Ezugwu EC, Agu PU. The incidence and management outcome of preterm premature rupture of membranes (PPROM) in a tertiary hospital in Nigeria. Am J Clini Med Res. 2014;2(1):14-7.

8. Revathi V, Sowjanya R, Lavanya S. Maternal and perinatal outcome in premature rupture of membranes at term. IOSR-JDMS. 2015;14:12-5.

9. Singhal S, Puri M, Gami N. An analysis of factors affecting the duration of latency period and its impact on neonatal outcome in patients with PPROM. Arch Gynecol Obstet. 2011;284(6):133943.

10. Kadikar GK, Gandhi MR. A study of fetomaternal outcome in premature rupture of membranes. 2014;3(3).
11. Lim JJ, Allen VM, Scott HM, Allen AC. Late preterm delivery in women with preterm prelabour rupture of membranes. J Obstet Gynaecol Canada. 2010;32(6):555-60.

12. Magee B, Smith G. Histological chorioamnionitis associated with preterm prelabour rupture of membranes at Kingston General Hospital: a practice audit. J Obstet Gynaecol Canada. 2013;35(12):10839.

13. Emechebe CI. Determinants and complications of pre-labour rupture of membranes (PROM) at the University of Calabar Teaching Hospital (UCTH), Calabar, Nigeria. Parity. 95:100-0.

Cite this article as: Diraviyam JMV, Karunakaran

L. Maternal and perinatal outcome in preterm premature rupture of membranes. Int J Reprod Contracept Obstet Gynecol 2017;6:2498-2502. 